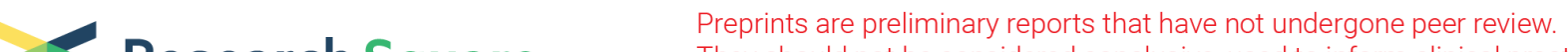 Research Square $\quad \begin{aligned} & \text { They should not be considered conclusive, used to inform clinical practice, } \\ & \text { or referenced by the media as validated information. }\end{aligned}$ \\ Profiling B cell immune responses to identify neutralizing antibodies from convalescent COVID- 19 patients
}

\section{Lisu Huang}

Xinhua Hospital, Shanghai Jiao Tong University School of Medicine, Shanghai, China.

\section{Bingqing Shen}

HiFiBiO (Shanghai) Co. Ltd, Shanghai, China.

\section{Yu Guo}

State Key Laboratory of Medicinal Chemical Biology, Nankai University, Tianjin, China.

\section{Shu Shen}

State Key Laboratory of Virology and National Virus Resource Center, Wuhan Institute of Virology, Chinese Academy of Sciences, Hubei, China.

\section{Heyu Huang}

Xinhua Hospital, Shanghai Jiao Tong University School of Medicine, Shanghai, China.

\section{Qiuling Xie}

Xinhua Hospital, Shanghai Jiao Tong University School of Medicine, Shanghai, China.

\section{Mingjie Chen}

HiFiBiO (Shanghai) Co. Ltd, Shanghai, China.

\section{Xinxin Zeng}

Xinhua Hospital, Shanghai Jiao Tong University School of Medicine, Shanghai, China.

\section{Shanshan Wang}

HiFiBiO (Shanghai) Co. Ltd, Shanghai, China.

\section{Dakun Huang}

Yongjia County Center for Disease Control and Prevention, Zhejiang, China.

\section{Mengshi Huang}

HiFiBiO (Shanghai) Co. Ltd, Shanghai, China.

\section{Yushu Qiu}

Xinhua Hospital, Shanghai Jiao Tong University School of Medicine, Shanghai, China.

\section{Xiaochen Han}

HiFiBiO (Shanghai) Co. Ltd, Shanghai, China.

\section{Qingqing Jing}

HiFiBiO (Shanghai) Co. Ltd, Shanghai, China.

\section{Minmin Lu}

HiFiBiO (Shanghai) Co. Ltd, Shanghai, China. 


\section{Chunjiang Pi}

HiFiBiO (Shanghai) Co. Ltd, Shanghai, China.

\section{Jia Wu}

HiFiBiO (Shanghai) Co. Ltd, Shanghai, China.

\section{Minfang Feng}

HiFiBiO (Shanghai) Co. Ltd, Shanghai, China.

\section{Ouyang Li}

HiFiBiO (Shanghai) Co. Ltd, Shanghai, China.

\section{Yun-Yueh Lu}

HiFiBiO (Shanghai) Co. Ltd, Shanghai, China.

\section{He Zhou}

HiFiBiO (Shanghai) Co. Ltd, Shanghai, China.

\section{Cheryl Cui}

Nest.Bio Labs, Zhejiang, China.

\section{Jeff He}

HiFiBiO (Shanghai) Co. Ltd, Shanghai, China.

\section{Shuming Pan}

Xinhua Hospital, Shanghai Jiao Tong University School of Medicine, Shanghai, China.

\section{Da Chen}

State Key Laboratory of Medicinal Chemical Biology, Nankai University, Tianjin, China.

\section{Xingdong Zhou}

State Key Laboratory of Medicinal Chemical Biology, Nankai University, Tianjin, China.

\section{Yuan Wang}

State Key Laboratory of Medicinal Chemical Biology, Nankai University, Tianjin, China.

\section{Yujie Tian}

State Key Laboratory of Medicinal Chemical Biology, Nankai University, Tianjin, China.

\section{Wei Wang}

Shanghai Institute for Advanced Immunochemical Studies, ShanghaiTech University, Shanghai, China.

\section{Wanju Zhang}

Shanghai Public Health Clinical Center, Fudan University, Shanghai, China.

\section{Fei Deng}

State Key Laboratory of Virology and National Virus Resource Center, Wuhan Institute of Virology, Chinese Academy of Sciences, Hubei, China.

\section{Liang Schweizer ( $\nabla$ l.schweizer@hifibio.com )}

HiFiBiO (Shanghai) Co. Ltd, Shanghai, China.

\section{Hongkai Zhang ( $\square$ hongkai@nankai.edu.cn )}

State Key Laboratory of Medicinal Chemical Biology, Nankai University, Tianjin, China.

\section{Kun Sun ( $\nabla$ sunkun@xinhuamed.com.cn )}

Xinhua Hospital, Shanghai Jiao Tong University School of Medicine, Shanghai, China. 


\section{Research Article}

Keywords: SARS-CoV-2, neutralizing antibody, single-cell sequencing

Posted Date: August 12th, 2020

DOl: https://doi.org/10.21203/rs.3.rs-38173/v2

License: (c) (i) This work is licensed under a Creative Commons Attribution 4.0 International License. Read Full License 


\section{Abstract}

The pandemic Coronavirus Disease 2019 (COVID-19) causes noticeable morbidity and mortality worldwide. In addition to vaccine and antiviral drug therapy, the use of severe acute respiratory syndrome coronavirus-2 (SARS-CoV-2) neutralizing antibodies for treatment purposes is a viable alternative. In this study, we aimed to profile the humoral responses and identify neutralizing antibodies against SARS-CoV2 using high-throughput single-cell sequencing that tailored to B cell receptor sequencing. From two convalescent patients with high serum titer against SARS-COV-2, we identified seven antibodies specifically binding to SARS-CoV-2. Among these, the most potent antibody, P4A1 was demonstrated to block the binding of spike protein to its receptor angiotensin-converting enzyme 2 (ACE2), and prevent the viral infection in neutralization assays with pseudovirus as well as live virus at $\mathrm{nM}$ to sub-nM range. Moreover, antibody P4A1 can also bind strongly to spike protein with N354D/D364Y, R408I, W436R, V367F or D614G mutations respectively, suggesting that the antibody alone or in combination with other antibodies that recognize different variations of SARS-CoV-2, may provide a broad spectrum therapeutic option for COVID-19 patients.

Authors Lisu Huang, Bingqing Shen, Yu Guo, and Shu Shen contributed equally to this work.

\section{Introduction}

The Coronavirus Disease 2019 (COVID-19) caused by the novel SARS-CoV-2 coronavirus, which belongs to lineage $B$ of the betacoronavirus genus, Coronaviridae, has become a global public health crisis $^{1,2}$. A better understanding of the pathological mechanisms caused by the virus and development of new therapeutic agents are urgently needed. Since the emergency of the pandemic, significant progress has been made to characterize the virus ${ }^{1,3,4}$. Several marketed products and molecules in clinical trial such as remdesivir are under clinical investigation to treat COVID-19. Meanwhile, convalescent plasma (CP) transfusion therapy and plasma derived-therapies have been demonstrated as effective options for COVID-19 to reduce the viral load, and improve clinical manifestations and laboratory parameters ${ }^{5,6}$. Nevertheless, one caveat to CP transfusion therapy is that stable plasma supply from the recovered patients is limited, and the quality for such supply is hard to control. Inevitably, the risks accompanied with plasma transfusion, such as non-neutralizing antibody-dependent enhancement of infection and allergic transfusion reactions, raise the major concern in the application of such therapy.

The spike (S) glycoprotein on the surface of SARS-CoV-2 mediates ACE2 receptor recognition of the virus and membrane fusion for infection of host cells. The receptor binding domain (RBD) of the $S$ protein is responsible to the binding to ACE2 ${ }^{7,8}$. Therefore, it is the primary target for neutralizing antibody to prevent the binding of the virus to its host receptor. Neutralizing antibodies could be a promising intervention to COVID-19 due to their therapeutic effectiveness and the capability for quality manufacturing. Sera from convalescent patients provide a source of antiviral neutralizing antibodies that conferring protective immunity on recipients, as was seen with antibodies identified from patients infected with the Ebola virus ${ }^{9,10}$. 
To identify antibodies from convalescent COVID-19 patients, memory B cells specific for SARS-CoV-2 S protein were isolated from peripheral blood mononuclear cell (PBMC) by fluorescence activated cell sorting (FACS), and neutralizing antibodies were individually identified by RT-PCR from the sorted cells ${ }^{11}$, $12,13,14$. Nevertheless, this method is confined by the limited throughput. The advent of high throughput single cell sequencing technology demonstrated the potential for obtaining VH-VL pairing information in a high-throughput manner ${ }^{15,16}$. The latest generation of single cell RNA sequencing barcoding technologies developed by companies using droplets (such as 10×Genomics or BioRad) or microfluidics arrays (such as BD Rhapsody or Singleron) can be utilized to identify $V$ gene sequences of a cohort of sorted B cells such as selected B cells using FACS. Indeed, Cao et al identified potent SARS-COV-2 neutralizing antibodies by high-throughput single cell RNA and VDJ sequencing of antigen enriched $B$ cells from convalescent patients using 10×Genomics Chromium platform ${ }^{17}$.

In this report, we mined the immune repertoires of patients recovered from COVID-19 with our single-cell sequencing platform that tailored to sequence human BCR, and identified novel antiviral antibodies that might be potential therapeutic agents. The neutralizing antibodies exhibited potent anti-viral activities and expected pharmacological profiles. In addition, the lead antibody demonstrated activities against multiple variants of the $S$ protein and was well positioned for multivalent as well as combination therapies.

\section{Results}

\section{Patient blood sample collection and characterization}

Among the 23 patients, there are 14 males (Supplementary Table 1). The medium age is 47.0 (Q1-Q3: 31.0-51.0) years old. Most of them had the symptom of fever and cough. Only one patient developed abnormal heart function and four patients had higher liver enzymes. The total rate of severe cases was $17.4 \%$ in our study population according to Chinese COVID-19 Guideline, and no deaths occurred.

To characterize the antiviral immune responses, antibody titers in these patient serums were determined by ELISA against the SARS-CoV-2 S protein. Sera from convalescent patients clearly showed higher titers as compared to sera from healthy donors (Fig. 1a). Sera were further evaluated for binding to the nucleocapsid (N) protein of SARS-CoV-2 (Fig. 1a). The relative reactivity of patient sera against the $S$ protein correlates well with that against the $\mathrm{N}$ protein.

The spike S protein is presented as a trimer on the viral envelope and consists of two subunits: a receptorbinding S1 subunit on top of a S2 stalk that mediates membrane fusion and viral entry ${ }^{18}$. To assess the immune response to different domains of the SARS-CoV-2S protein, serum from the patient 4 who exhibited the highest titer against $\mathrm{S}$ protein was titrated against full-length $\mathrm{S} 1+\mathrm{S} 2$-His protein, $\mathrm{S} 1-\mathrm{mFc}$ fusion protein, S1-His protein, and the S1 receptor binding domain (S1-RBD). Binding activity was 
stronger against the full-length $\mathrm{S} 1+\mathrm{S} 2-\mathrm{His}$ protein and the $\mathrm{S} 1-\mathrm{mFc}$ fusion protein than the monomeric S1His and S1-RBD domains (Fig. 1b), suggesting that an immune response is also generated against the S2 domain and that the oligomerization of the $S$ protein influences antibody binding.

To characterize the B cell populations of patient samples, PBMCs of two patients with the highest serum titer (the patient 4 and the patient 20) and one healthy donor were analyzed using flow cytometry. Cells were stained with a panel of anti-CD 19/CD27/CD38 antibodies, and plasmablasts (characterized by $\mathrm{CD} 19^{+} / \mathrm{CD} 27^{+} / \mathrm{CD} 38^{+}$) and memory B cells (characterized by $\left.\mathrm{CD} 19^{+} / \mathrm{CD} 27^{+} / \mathrm{CD} 38^{-}\right)$were identified. While total CD19 positive populations from two patients are somewhat lower than the control healthy donor with overall reduced lymphocytes as reported previously ${ }^{19}$, the fraction of memory B cells (CD27 $7^{\text {high }}$ CD38 ${ }^{\text {low }}$ ) are significantly higher in the two convalescent patient samples than in the healthy donor control and the percentages of plasmablasts $\left(C D 27^{\text {high }} \operatorname{CD} 38^{\text {high }}\right)$ are more than 20-fold higher as compared with healthy donor sample (Fig. 2).

An increased fraction of memory B cells in convalescent patients, which indicated that the humoral immune responses play an important role in natural SARS-CoV-2 viral clearance and may serve as a potential complementary diagnostic tool in clinical settings, were observed ${ }^{9}$.

\section{High-throughput single-cell B-cell receptor sequencing}

To initiate barcoded single-cell reverse transcription, B cells were compartmentalized in droplet together with lysis buffer, reverse transcriptase and a hydrogel beads using the microfluidics chip for droplet generation. Each hydrogel bead carried VH and VL specific primers tagged with a unique barcode which can specifically reverse transcribe VH and VL mRNA from individual B cells. Subsequently, the VH and VL regions were amplified by nest PCR, where the cell barcodes were retained during the whole sequencing library preparation process. A dedicated bioinformatics pipeline was developed for analysis of the single cell antibody sequencing result and the cognate $\mathrm{VH}$ and $\mathrm{VL}$ of the same $\mathrm{B}$ cell can be identified based on the barcode information (Fig. 3).

To validate the paring efficiency and accuracy of this single cell sequencing procedure, we profiled two hybridoma cell lines (9E10 and $\mathrm{H} 2$ ) mixed at a 9E10/H2 ratio of 100:1. Post-sequencing analysis demonstrated that $98 \%$ paired barcodes were unambiguously mapped to one of the cell lines with correctly paired VH and VL. The proportion of each cell line detected was consist with the initial proportion of the cells $(2.5 \% \mathrm{H} 2)$, reflecting the robustness of this approach.

Next, we set out to isolate SARS-COV-2 neutralizing antibody from 2 convalescent patients (the patient 4 and the patient 20). We enriched SARS-CoV-2 S protein binding B cells from PBMC of convalescent patients with magnetic beads that conjugated with biotinylated $S$ protein as probes. The enriched $B$ cells were individually compartmentalized in droplet together with lysis buffer, reverse transcriptase and a 
hydrogel beads to prepare sequencing library. We sequenced the library using Illumina Miseq PE 300 to ensure the full-length recovery of antibody variable gene fragments.

Nine and thirteen antibodies were identified from the patient 4 and the patient 20, respectively. Six antibody sequences were filtered out for they contained critical liability motifs (such as additional cysteine located in the CDR3 and glycosylation site anywhere in the variable region) that would make them extremely difficult to develop into a drug. The remaining sixteen antibodies were subject to further characterization.

\section{Characterization of SARS-CoV-2 S protein specific antibodies}

To confirm the function of the antibodies, their genes were synthesized and the recombinant antibodies were expressed and purified to evaluate their binding to the full-length S protein. ELISA results showed that 7 antibodies bound to the full length S protein of SARS-CoV-2, among which P4A1 was the strongest binder (Fig. 4a). Interestingly, when their binding to the specific domains of $S$ protein is evaluated, P4A1, P4A2, P20A2 and P20A3 were shown to be binders to S1 fragment, while P20A1 bound to $S 2$ fragment (Fig. 4a), respectively.

We further examined the blocking effects of the five strong binders on the binding of S1 to Vero E6 cell line by flow cytometry analysis (Fig. 4b). P4A1, P4A2, P20A2 and P20A3 can block S1 binding to Vero E6 at the $\mathrm{nM}$ range while $\mathrm{S} 2$ binder P20A1 does not affect S1 binding to the receptor on Vero E6 cells as expected. Next, we studied the neutralizing activities of antibodies against HIV-vectored pseudovirus. Antibodies P4A1 exhibited potent neutralizing activity, with $\mathrm{IC}_{50}$ of $0.53 \mathrm{nM}$. P20A2 and P20A3 showed $\mathrm{IC}_{50} \mathrm{~S}$ at $5.80 \mathrm{nM}$ and $4.07 \mathrm{nM}$ respectively (Fig. $4 \mathrm{c}$ ). These antibody activities from $\mathrm{S}$ protein binding to blockage of S1 binding to Vero E6 cells and further to pseudovirus neutralization suggested that S1 binding is a critical step for virus to bind to host cells, and blocking of S1 binding to host cells could potentially be interpreted as anti-viral activity.

\section{Bioinformatically identification of somatic variants of antibody P4A1}

Since somatic hypermutation does not distinguish between favorable and unfavorable mutations, we searched heavy chains in the NGS results based on similarity to P4A1 heavy chain. Eleven P4A1-class sequences were bioinformatically identified. When reconstituted with the light chain from P4A1, antibodies demonstrated various levels of binding abilities to the full length $S$ protein as well as abilities to block the S1 protein binding to human ACE2 receptor (Fig. 5a, 5b). A few closely related antibodies 


\section{Neutralization activity of antibodies against live SARS-CoV-2}

The neutralizing activities of the lead antibodies, P4A1, P20A2 and P20A3 were further confirmed in live SARS-CoV-2 virus entry assay. Vero E6 cells were infected with SARS-CoV-2 virus at $100 \mathrm{TCID}_{50}$ in the presence of the antibodies at different concentrations. Using fluorescent labeled nuclear protein of SARCoV-2, infected cells can be observed by florescence microscopy. Antibody P4A1 demonstrated strong neutralization against SARS-CoV-2, which is more than $50 \%$ inhibition at $6.5 \mathrm{nM}$, while P20A2 demonstrated $74 \%$ inhibition at $62.7 \mathrm{nM}$, and P20A3 showed 93.8\% inhibition at 50.2nM (Fig. 6a). However, antibody P20A1 did not have any neutralization against SARS-CoV-2 (Fig. 6a). The inhibition effect of these antibodies was further evaluated by calculating $\mathrm{IC}_{50}$ and $\mathrm{IC}_{90}$. Antibody P4A1 showed IC 50 of $2.724 \mathrm{nM}$ and IC 90 of $7.07 \mathrm{nM}$. IC $\mathrm{I}_{50}$ and $\mathrm{IC}_{90}$ of P20A2 are $34.23 \mathrm{nM}$ and $98.90 \mathrm{nM}$, respectively; and $\mathrm{IC}_{50}$ and $\mathrm{IC}_{90}$ of $\mathrm{P} 20 \mathrm{~A} 3$ are $29.07 \mathrm{nM}$ and $45.20 \mathrm{nM}$, respectively (Fig. 6b).

\section{Cross-reactivity against SARS-CoV-2 S protein mutations}

As the pandemics progressed, the mutations accumulated in S protein and conferred selective advantages in transmission and resistance to the antibody intervention. Among the mutations, the spike protein mutation D614G is of urgent concern. It began spreading in Europe in February and rapidly became the dominant virus strain in many countries ${ }^{20}$. Therefore, we preventatively examined the affinity of P4A1 with RBD protein of 4 mutation strains and S1 protein with D614G mutation by using surface plasma resonance (SPR). As shown in Figure 7, antibody P4A1 can strongly bound to all mutations with $\mathrm{Kd}$ ranging from $10^{-9}$ to $10^{-14} \mathrm{M}$. The antibody affinities for protein with mutation N354D/D364Y, W436R or V367F are too high to be measured accurately. The results indicated that antibody P4A1 could potentially have broad cross-neutralization effects against SARS-CoV-2 mutation strains.

\section{Discussion}

A deeper understanding of the immune response mounted by patients recovering from COVID-19 may give insight into disease epidemiology and inform the development of novel therapies. Here, we observed that the levels of circulating plasma cells and memory B cells increased in convalescent patients, weeks after initial infection and days after recovery, suggesting that the humoral immune response is critical in eliminating viruses. The relationship of viral load, replication capability, antiviral immune responses, and disease severity has been of tremendous interest for future study. Convalescent patient sera have been 
applied as an emergent therapeutic strategy to transfer protective immunity to newly diagnosed patients and at-risk populations. The neutralizing antibodies identified from these sera provide potential to scale up for COVID-19 treatment. Further mining of immune repertoires from patients recovering from COVID19 will be of importance and significance to control the pandemic.

With single-cell sequencing platform, we analyzed the immune repertoires of two convalescent COVID-19 patients and identified antibodies binding to SARS-CoV-2 coronavirus. One antibody P4A1 demonstrated potent SARS-CoV-2-specific neutralization activity in vitro. Moreover, the antibody displayed broad reactivity to emerging $S$ protein mutations. Combination of antibodies such as P4A1 with a S2 binder such as P20A1 with no direct neutralizing activity, yet could affect other virus function such as viral entry, or combinations with neutralizing antibodies targeting nonoverlapping epitopes are worth to explore. It is also interesting to study what type of therapies would be the most effective way to prevent the escaping mutations of the virus.

In conclusion, our lead neutralizing antibody P4A1, isolated from convalescent COVID-19 patients, could be developed as prophylactic and therapeutic intervention for COVID-19. Furthermore, our single-cell BCR sequencing approach can be extended to pursue fully human therapeutic monoclonal antibodies from vaccinated and naturally infected individuals, that may provide additional therapeutic options to broad spectrum of the coronavirus.

\section{Materials And Methods}

\section{Study design and participants}

We recruited patients between February $11^{\text {th }}, 2020$ and March 1 $1^{\text {st }}, 2020$ at Yongjia People's Hospital and Yongjia Center for Disease Control and Prevention in Zhejiang Province, China. All the patients with COVID-19 were clinically diagnosed and laboratory-confirmed. A laboratory-confirmed case of COVID-19 was defined as a positive result on high-throughput sequencing or real-time reverse-transcriptase polymerase-chain-reaction (RT-PCR) assay of nasal and pharyngeal swab specimens on the basis of the WHO interim guidance (WHO, 2020). The criteria of recovery were normal temperature for at least 3 days, obvious improvement in clinical symptoms, significant absorption of pulmonary inflammation on computer tomography scan and negative tests for SARS-CoV-2 two times in a row with a test interval for at least one day. Patients tested positive for HIV, HBV, HAV, HCV or syphilis were excluded. All the clinical data were all reviewed by a trained team of physicians from department of respiratory, intensive care and infectious diseases clinicians. Patients without the baseline assessment test were excluded. 23 patients and two healthy volunteers were included in the samples. The sample size is sufficient to find blood with high antibody titer against the SARS-CoV-2 S protein for the subsequent antibody isolation.

Blood samples were collected and peripheral blood mononuclear cells (PBMCs) and plasma were isolated and frozen for subsequent analysis between 3-4 days after patients recovered from COVID-19. The plasma was titered against the SARS-CoV-2 antigens and two samples with the highest titer were 
chosen for further analysis. The study was approved by the Ethics Committee of Xinhua Hospital affiliated to Shanghai Jiao Tong university, Yongjia People's Hospital and Yongjia Center for Disease Control and Prevention in Zhejiang Province. A written informed consent was achieved from patients or their legal guardian.

\section{PBMC and serum sample preparation}

Blood samples were collected 3-4 days after donors were discharged from the hospital and separated into plasma and peripheral blood mononuclear cells (PBMCs) by Ficoll-Hypaque gradient centrifugation. Plasmas and PBMCs were maintained in freezing media and stored at $-80^{\circ} \mathrm{C}$. The plasma was heatinactivated at $56^{\circ} \mathrm{C}$ for $1 \mathrm{~h}$ before use.

\section{Titer measurements by ELISA}

The ELISA protocol was adapted from previously established protocols. 384 well plates were coated overnight at $4^{\circ} \mathrm{C}$ with PBS containing $1 \mu \mathrm{g} / \mathrm{mL}$ of the respective protein S1 RBD-mFc tag (SinoBiological, catalog\#40592-V05H) orFull length S-his (SinoBiological, catalog\#40589-V08B1) orS1-mFc

(Sinobiological, catalog\#40591-V05H1 or S1-his (Kactus, catalog\# COV-VM4S1). The next day the plate was washed 4 times with washing buffer (PBS and $0.05 \%$ Tween) and then incubated 1 hour at $37^{\circ} \mathrm{C}$ in blocking buffer (PBS with 2\% BSA). After two washes the plate was incubated for 1 hour at $37^{\circ} \mathrm{C}$ with the serum or the positive control ACE2 protein (Sinobiological,catalog\#10108-H08H). The human serum samples were diluted to 1:100 in PBS $+2 \%$ BSA followed by 5 -fold serial dilutions. The plates were then washed 4 times and incubated for 1 hour in blocking buffer (PBS with $0.05 \%$ Tween and $1 \%$ BSA) containing diluted (1:5000) secondary antibody (HRP labeled mouse anti-human IgG Fc antibody, Thermo Fisher, catalog\#05-4220, RRID: AB_ 2532922, clone name: HP6017, Lot number\# UC282110) for 1 hour at room temperature. Following this the plate was washed again 4 times and developed in TMB substrate (Biolegend, catalog\#421101) for 5 min before stopping the reaction with the stop solution (Solarbio,catalog\# C1058).

\section{Flow cytometry for B cell immune profiling}

PBMC was thawed at $37^{\circ} \mathrm{C}$ and then centrifuged at $450 \mathrm{~g}$ for $8 \mathrm{~min}$. The supernatant was discarded, and the cells resuspended in $200 \mu \mathrm{L}$ of DMEM (Gibco, catalog\#11995-065). Following the addition of $1 \mu \mathrm{L}$ of Dnase I (Qiagen, catalog\#79254), cells were incubated for 3 min and spun down again. The pellet was resuspended in 20 $\mu \mathrm{L}$ of FcR Blocking Reagent (Miltenyi Biotec, catalog\#130-059-901), incubated for 10 mins and centrifuged. The cells were suspended in 200 $\mathrm{L}$ PBS. $3 \mu \mathrm{L}$ (1:70 dilution) of anti-CD19 (FITC labeled, eBioscience, catalog\#11-0199-42, RRID: AB_AB_10669461, clone name: HIB19, Lot number\#1995396), anti-CD27 (APC labeled, eBiosciences, catalog\#17-0279-42, RRID: AB_10671130, 
clone name: 0323, Lot number\#2087761), anti-CD38 (PE labeled mouse IgG1 isotype control, eBioscience, catalog\#12-0388-42, RRID: 1989502, clone name: HB7, Lot number\#1989502) or its isotype PE Mouse isotype control (BioLegend, catalog\#400114, clone name: MOPC-21, Lot number\#4307319) and FITC labeled mouse lgG1 isotype control (ebioscience, catalog\#11-4714-41, RRID: AB_10598647, clone name: P3.6.2.8.1, Lot number\#4307319) and APC labeled mouse IgG1 isotype control ( BD Biosciences, catalog\#550854, clone name: MOPC-21, Lot number\#7215834) was then added, and incubated for $30 \mathrm{~min}$ at room temperature. Following centrifugation, cells were resuspended in a $100 \mu \mathrm{L}$ of $4 \%$ PFA (Beyotime, catalog\#P0099-500ml). After 10 min the cells were washed twice by centrifugation and finally resuspended in PBS and ready for flow cytometry analysis using a Cytoflex(Beckman Coulter). The median fluorescence intensity (MFI) was calculated with FlowJo (version 10.6.0).

\section{Patient sample information for FACS}

\begin{tabular}{|l|l|l|l|l|}
\hline & labeling & Cell Number & Viability (\%) & Gender \\
\hline PBMC & Patient 4 & $2.54 \times 10^{5} / \mathrm{mL}, 200 \mu \mathrm{L}$ & 84 & Female \\
\hline PBMC & Patient 20 & $7.1 \times 10^{5} / \mathrm{mL}, 200 \mu \mathrm{L}$ & 90 & Male \\
\hline PBMC & Healthy donor & $7.0 \times 10^{5} / \mathrm{mL}, 200 \mu \mathrm{L}$ & 92 & Male \\
\hline
\end{tabular}

\section{Isolation of S protein specific B cells}

Avitag and His tag SARS-CoV-2 Sprotein was expressed in human embryonic kidney cell 293-F (ThermoFisher, catalog\# R79007. The cell line was not authenticated.) which was tested negative for mycoplasma contamination. The $S$ protein was purified using HISTRAP HP column(GE, catalog\#175248-01) and biotinylated using Biotin-Protein Ligase (GeneCopoeia, catalog\#BI001). The B cells were stained with biotinylated $S$ protein and incubated at $4^{\circ} \mathrm{C}$ for 1 hour. After incubation, the cells were washed three times with PBS. Subsequently, the cells were labeled with Streptavidin microbeads (Miltenyi Biotec, catalog\# 130-048-101) at $4^{\circ} \mathrm{C}$ for 1 hour. After the incubation, the cell suspension was loaded onto a MACS column which is placed on a magnetic field of a MACS separator. The column was washed three times so the magnetically labeled B cells was retained in the column and unlabeled cells passed through. After removing from the MACS separator, the magnetically labeled B cells were eluted. The isolated B cells were counted by using $0.4 \%(w / v)$ Trypan blue stain.

\section{Single-cell BCR sequencing}


Enriched S protein specific B cells were individually co-compartmentalized in droplets with single barcoded hydrogel beads and lysis and reverse transcription reagents using a microfluidic device as described ${ }^{15}$. Droplets of $\sim 1 \mathrm{~nL}$ volume were formed at $250 \mathrm{~s}-1$. The droplets were collected in a $1.5 \mathrm{~mL}$ tube containing HFE-7500 (3M ${ }^{\mathrm{TM}}$, catalog\#Novec 7500 ) and $0.1 \%$ surfactant (RanBio, catalog\#008FluoroSurfactant), UV photo-cleaved for 90 seconds (OmniCure ac475-365) and incubated at $50^{\circ} \mathrm{C}$ for cell lysis and cDNA synthesis. Reverse transcription of $\mathrm{VH}$ and $\mathrm{VL}$ mRNAs from single B cells took place in droplet using barcoded primers carrying the T7-SBS12 sequences followed by barcode and genespecific primer sequences complementary to heavy chain $\mathrm{J}$ genes and light chain constant region sequences.

The emulsion containing the barcoded cDNA was broken by adding one volume of $1 \mathrm{H}, 1 \mathrm{H}, 2 \mathrm{H}, 2 \mathrm{H}-$ Perfluoro-1-octanol (Sigma-Aldrich, catalog\#370533-25G) after the droplet RT reaction finished. The pooled, barcoded cDNAs were purified with Agencourt RNA CleanUp beads (Beckman, catalog\#A63987) at a 1:1 ratio ( $\mathrm{vol} / \mathrm{vol})$ twice and eluted in $60 \mu \mathrm{L}$ DNase- and RNase-free $\mathrm{H}_{2} \mathrm{O}$. The sequencing library was generated by two-step nested PCR using GoTaq Polymerase (Promega, catalog\#M7406). In the first PCR, forward primers were priming on the $\mathrm{T7}$ and with reverse primers priming on the $\mathrm{VH}, \mathrm{V} \lambda$ and $\mathrm{VK}_{\mathrm{K}}$ leader and framework 1 sequenced of the $V$ genes. In the second PCR, the forward primer appends the lllumina P7 and Illumina index sequences by priming on the SBS12 sequence and the reverse primer appends the Illumina P5 and SBS3 sequence. The approximately 550 bp final PCR products were extracted by agarose gel electrophoresis (Qiagen, catalog\#28606). The constructed NGS libraries were sent out for sequencing using Illumina MiSeq PE300 which allows sequencing of the entire VH and VL domain as well as the barcode sequence (GeneScript sequencing service supplier) with data varying from 6-12 million reads per samples. The resulting FASTQ data was analyzed by a bioinformatics pipeline enable trimming, merging, barcode extraction and clustering as described ${ }^{16}$. Briefly, paired-end reads were first trimmed at the $3^{\prime}$ end to remove low quality score bases then merged using the program FLASH requiring at least $10 \mathrm{bp}$ overlap. The barcodes were extracted form merged reads followed by clustering requiring the DNA sharing at least $93 \%$ identify. The consensus sequence was created from clusters by aligning up to 200 sequencing using ClustalO(version1.2), and each antibody sequence was characterized for immunoglobulin content using VDJFasta(version 2.0). We also applied a minimum number of reads 10 for $\mathrm{VH}$ and $\mathrm{VL}$. VH-VL pairing was carried out by identifying the most abundant $\mathrm{VH}$ and $\mathrm{VL}$ consensus sequence (by number of reads that contributed to that in each barcode cluster).

\section{Production of recombinant antibody}

The DNA of variable regions of the heavy and light chains were synthesized by Genewiz and cloned into expression plasmids containing the human IgG1 heavy chain and kappa light chain constant regions. The antibodies were expressed in 293F cell for 5 days after the co-transfection of both heavy and light chain expression plasmids. Antibodies were purified from cell culture supernatants using Protein-A affinity chromatography. 


\section{Antibody binding and competition with receptor ACE2}

The binding affinity of antibodies to $S$ protein was analyzed by ELISA. 384 well plate (Corning, catalog\#3700), was coated overnight at $4^{\circ} \mathrm{C}$ with PBS containing $30 \mu \mathrm{L} 20 \mathrm{nM}$ of the SARS-CoV-2 Spike S1+S2 ECD, his Tag protein (SinoBiological, catalog\#40589-V08B1). The next day the plate was washed 5 times with washing buffer (PBS and $0.05 \%$ Tween) and then incubated 1 hour at room temperature in blocking buffer (PBS with 2\% BSA). After 5 washes the plate was incubated with serial dilution of purified antibodies for 1 hour at room temperature. The plates were then washed 5 times and incubated for 1 hour in blocking buffer(PBS with $0.05 \%$ Tween and $1 \%$ BSA) containing Mouse anti-Human IgG Fc HRP labeled (Thermo Fisher, catalog\#05-4220, RRID: AB_ 2532922, clone name: HP6017, Lot number\# UC282110, dilution 1:5000) for 1 hour at room temperature. Following this the plate was washed again 5 times and developed in TMB substrate (Biolegend, catalog\#421101) for 5 min before stopping the reaction with the stop solution (Solarbio, catalog\#C1058). The OD values were determined using Thermo MultiSkan or MD SpectraMax i3X at 450nm wavelength, data was analyzed with GraphPad Prism (version 8.0.1).

The blocking with receptor ACE2 was performed using cell surface expressed ACE2. 10nM SARS-CoV-2 spike S1, mFc tag protein (SinoBiological, catalog\#40591-V05H1) was incubated with serial dilution of purified antibodies at room temperature for 1 hour and then added to Vero E6 cells (ATCC,CRL-1587. The cell line was validated by CoBIOER (http://www.co-bioer.com/) and tested negative for mycoplasma contamination. These cells exhibit cytopathic effects when infected with multiple virus. Plaques are also produced. $10^{5}$ cellsper well) in duplicate. Then detection reagent rabbit anti mouse IgG Fc-AF647 (Jackson ImmunoResearch, catalog\#315-606-046, RRID: AB_2340251, 1:800 dilution) was used. Halfmaximal inhibitory concentration $\left(\mathrm{IC}_{50}\right)$ of the evaluated antibodies was determined with flow cytometry (Beckman, Cytoflex) and FlowJo software (version 10.6.0) analysis.

\section{Antibody neutralization activity against pseudovirus}

Murine leukemia virus-based SARS-CoV-2 S pseudotyped virus were prepared by GenScript. Human embryonic kidney cell HEK293T cells (ATCC,catalog\# CRL-3216, the cell line was tested negative for mycoplasma contamination. The cell line was not authenticated.) were co-transfected with envelopexpressing plasmid for SARS-CoV-2 Spike protein and HIV-1 backbone plasmids expressing packaging proteins and luciferase reporter at a mass ratio of 1:3 using Polyethylenimine (PEI) (Polyscience, catalog\#23966-1) according to the manufacture's instruction. Forty-eight hours after transfection, the supernatant containing pseudovirus was harvested and filtered with micropore filter $(0.45 \mu \mathrm{m})$ followed by ultracentrifugation at $83,000 \times \mathrm{g}, 4^{\circ} \mathrm{C}$ for $2 \mathrm{~h}$. The pseudovirus pellet was resuspended in DPBS and stored at $-196^{\circ} \mathrm{C}$. The titer of pseudovirus was determined by p24 ELISA kit (Takara, catalog\# 632200) following the manufacture's instruction. 
As for target cell establishment, HeLa cells (human cervix carcinoma cell, ATCC,catalog\# CCL-2, the cell line was tested negative for mycoplasma contamination. The cell line was not authenticated.) were transduced with lentivirus containing codon optimized human ACE2 CDS sequence (Uniprot ID: Q9BYF1) followed by single clone selection. The expression of ACE2 was validated with Spike-ECD (GenScript, catalog\#Z03481).

Neutralization assay were performed by incubating pseudovirus with serial dilution of purified antibodies at room temperature for 1 hour (starting from $100 \mathrm{ug} / \mathrm{ml}, 3$ folds dilution for 8 points). ACE2 overexpression HeLa cells (approximately $8 \times 10^{4}$ per well) were cultured in DMEM containing $10 \%$ FBS, $1 \mu \mathrm{g} / \mathrm{mL}$ puromycin were added in triplicate into virus-antibody mixture. Following infection at $37^{\circ} \mathrm{C}$ $5 \% \mathrm{CO} 2$ for 48 hours, luciferase activity was determined using Promega Bio-Glo luciferase assay (Promega, catalog\#G7491) system. The dose-response curves were plotted with the relative luminescence unit against the sample concentration. The assay results were processed by Microsoft Office Excel 2016 and GraphPad Prism 6. GenScript has applied this peudovirus neutralization assay to many other SARS-COV-2 neutralizing antibodies.

\section{Live virus assay}

Microneutralization assays towards live virus were performed as previously described ${ }^{1}$ with slight modifications. This Vero E6 cell line (ATCC, CRL-1586, the clone is different from the one used for in vitro blocking. The cell line was not authenticated) have been tested negative for mycoplasma contamination using a commercial EZ-PCR ${ }^{\mathrm{TM}}$ Mycoplasma Test kit (Biological Industries, Beit-Haemek, Israel; 20-70020; Lot\#: 1251719) (data not shown) and have been used for culture of the live virus ${ }^{1}$. Briefly, Vero E6 cells seeded in 96-well plates were incubated with SARS-CoV-2 (100 TCID 50 per well) (BetaCoV/Wuhan/WIV04/2019; National Virus Resource Center, Wuhan, China; IVCAS 6.7512) in absence or presence of diluted antibodies for 1 hour, fixed with 4\% paraformaldehyde diluted in PBS for 15 minutes at room temperature followed by penetrating solution containing $0.25 \%$ triton-X 100 for $10-15$ minutes. After three washes, cells were blocked at $37{ }^{\circ} \mathrm{C}$ for 1 hour using PBS containing $5 \%$ BSA, then incubated with in-house prepared anti-SARS-CoV-2 NP rabbit serum (1:1000 dilution) as primary antibody and Goat Anti-Rabbit IgG H\&L (Alexa Fluor® 488) (1:500 dilution, Abcam, Cambridge, UK; ab150077; Lot\#: GR3244688-2) as the secondary antibody. Specificity of the primary antibody to SARCoV-2 NP was validated by Western Blot with pCAGGS-SARS-CoV-2 NP transfected cells (data not shown). Cell nuclei were strained using Hoechst 33258 (Beyotime, Shanghai, China; C1018) at room temperature for 10 minutes. Images were taken using an Operetta CLSTM system (PerkinElmer, Waltham, USA), which counts numbers of nuclei and cells infected with viruses, respectively. Inhibition was calculated by the number of non-infected cells over the number of total nuclei. IC $\mathrm{C}_{50}$ and $\mathrm{IC}_{90}$ were calculated with GraphPad Prism 8.0. 


\section{Surface plasmon resonance analysis.}

SPR experiments were performed using Biacore T200 system (GE Healthcare). In brief, experiments were performed at $25^{\circ} \mathrm{C}$ in HBS-EP+ buffer. Antibody was immobilized onto a protein A sensor chip (GE healthcare, catalog\#29139131-AA). Serially diluted SARS-CoV-2 RBD (WT, AcroBiosystems, catalog\#SPD-C52H3), RBD (N354D/D364Y, AcroBiosystems, catalog\#SPD-S52H3), RBD (R408I, AcroBiosystems, catalog\#SPD-S52H8), RBD (W436R, AcroBiosystems, catalog\#SPD-S52H7), RBD (V367F, AcroBiosystems, catalog\#SPD-S52H4)or SARS-CoV-2 spike S1 domain (D614G, Sino Biological, catalog\#40591-V08H3) were injected through flow cells for 60 s of association followed by a $150 \mathrm{~s}$ dissociation phase at a flow rate of $30 \mu \mathrm{L} \mathrm{min}^{-1}$. Prior to next cycle, the sensor surface was regenerated with Glycine- $\mathrm{HCl}(\mathrm{pH} 1.5)$ for 30 s at a flow rate of $30 \mu \mathrm{L} \mathrm{min}{ }^{-1} . \mathrm{K}_{\mathrm{D}}$ values were calculated using the 1:1 binding kinetics model.

For all the experiments described above, the researchers were not blinded to group allocation during data collection and analysis. The experiments of Figures 4,5 and 6 were successfully replicated twice. The experiment of immune profiling of B cells of COVID-19 patients by flow cytometry was not replicated because the very limited amount of blood samples. All the detailed information,validation statements and relevant citations of primary antibodies can be found on the manufacturer's website of the supplier and Certificates of the antibodies can be searched by the lot numbers.

\section{Declarations}

\section{Acknowledgements}

We thank Dr Youjia Cao (Nankai University) for her suggestions on the manuscript, and Ning Zhang from Red Avenue Foundation for her support. The project is completed by National Biosafety Laboratory, Wuhan, Chinese Academy of Sciences. We are particularly grateful to the running team of the laboratory for their work. This work was supported by National key Research and Development plan of China

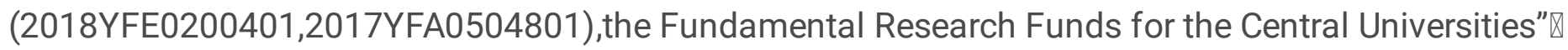
Nankai University(63201101 and ZB19100123), Industrial Cross Research Foundation of Shanghai Jiao Tong University (YG2020YQ27), Shanghai Municipal Health Commission (2017YQ033) and Shanghai Municipal Human Resources and Social Security Bureau (2018003) and HiFiBiO Therapeutics.

\section{Author contributions}

L. S., H. Z., F. D. and K. S. designed the study and wrote the manuscript. C. C. and H. Z. J. H. coordinated the study. L. H., B. S.,Y. G.,S. S., H. H., Q. X., M. C., X.Z, S. W., D. H., M. H., Y. Q., X. H., C. Y., Q.J, M. L., C. P., J. W., M. F., Y. L., S.P, D.C., X. Z. and Y. W. conducted the experiments. Y. T. and W. W. contributed to the figures. 


\section{Data availability}

The source data of Figures 1, 2, 4, 5a, 5b, 6b and 7 in this study are provided within this paper (supplementary zip file). Patent application has been filed on the reported antibodies(PCT/CN2020/096371 and PCT/CN2020/096360). All reagents and information presented in this study are available from corresponding authors upon reasonable request.

\section{References}

1.Zhou $\mathrm{P}$, et al. A pneumonia outbreak associated with a new coronavirus of probable bat origin. Nature 579, 270-273 (2020).

2.Zhu N, et al. A Novel Coronavirus from Patients with Pneumonia in China,2019. The New England journal of medicine 382, 727-733 (2020).

3.Diao B, et al. Reduction and Functional Exhaustion of T Cells in Patients With Coronavirus Disease 2019 (COVID-19). Frontiers in immunology 11, 827 (2020).

4.Walls AC, Park YJ, Tortorici MA, Wall A, McGuire AT, Veesler D. Structure, Function, and Antigenicity of the SARS-CoV-2 Spike Glycoprotein. Cell 181, 281-292 e286 (2020).

5.Zeng F, Chen X, Deng G. Convalescent plasma for patients with COVID-19. Proceedings of the National Academy of Sciences of the United States of America 117, 12528 (2020).

6.Shen C, et al. Treatment of 5 Critically III Patients With COVID-19 With Convalescent Plasma. JAMA $323,1582-1589$ (2020).

7.Wang Q, et al. Structural and Functional Basis of SARS-CoV-2 Entry by Using Human ACE2. Cell 181, 894-904 e899 (2020).

8.Wrapp D, et al. Cryo-EM structure of the 2019-nCoV spike in the prefusion conformation. Science 367, 1260-1263 (2020).

9.Casadevall A, Pirofski LA. The convalescent sera option for containing COVID-19. The Journal of clinical investigation 130, 1545-1548 (2020).

10.Bornholdt ZA, et al. Isolation of potent neutralizing antibodies from a survivor of the 2014 Ebola virus outbreak. Science 351, 1078-1083 (2016).

11.Chi $\mathrm{X}$, et al. A potent neutralizing human antibody reveals the $\mathrm{N}$-terminal domain of the Spike protein of SARS-CoV-2 as a site of vulnerability. bioRxiv, (2020).

12.Wu Y, et al. A noncompeting pair of human neutralizing antibodies block COVID-19 virus binding to its receptor ACE2. Science 368, 1274-1278 (2020). 
13.Robbiani DF, et al. Convergent Antibody Responses to SARS-CoV-2 Infection in Convalescent Individuals. bioRxiv, (2020).

14.Shi R, et al. A human neutralizing antibody targets the receptor binding site of SARS-CoV-2. Nature, (2020).

15.Goldstein LD, et al. Massively parallel single-cell B-cell receptor sequencing enables rapid discovery of diverse antigen-reactive antibodies. Commun Biol 2, 304 (2019).

16.Gérard A, et al. High-throughput single-cell activity-based screening and sequencing of antibodies using droplet microfluidics. Nature Biotechnology 38, 715-721 (2020).

17.Cao Y, et al. Potent neutralizing antibodies against SARS-CoV-2 identified by high-throughput singlecell sequencing of convalescent patients' B cells. Cell, (2020).

18.Li F. Structure, Function, and Evolution of Coronavirus Spike Proteins. Annu Rev Virol 3, 237-261 (2016).

19.Tian X, et al. Potent binding of 2019 novel coronavirus spike protein by a SARS coronavirus-specific human monoclonal antibody. Emerging microbes \& infections 9, 382-385 (2020).

20.Korber B, et al. Spike mutation pipeline reveals the emergence of a more transmissible form of SARSCoV-2. bioRxiv, (2020).

\section{Figures}



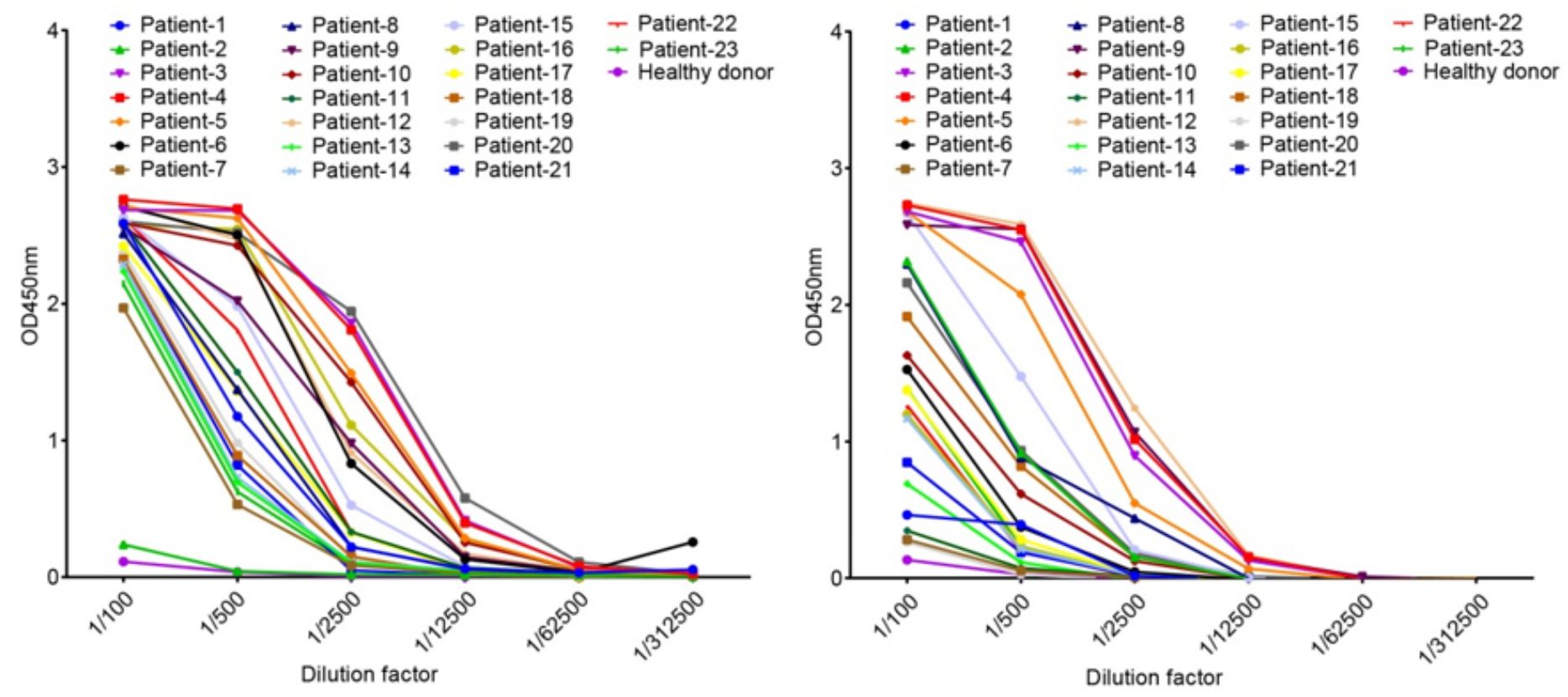

b

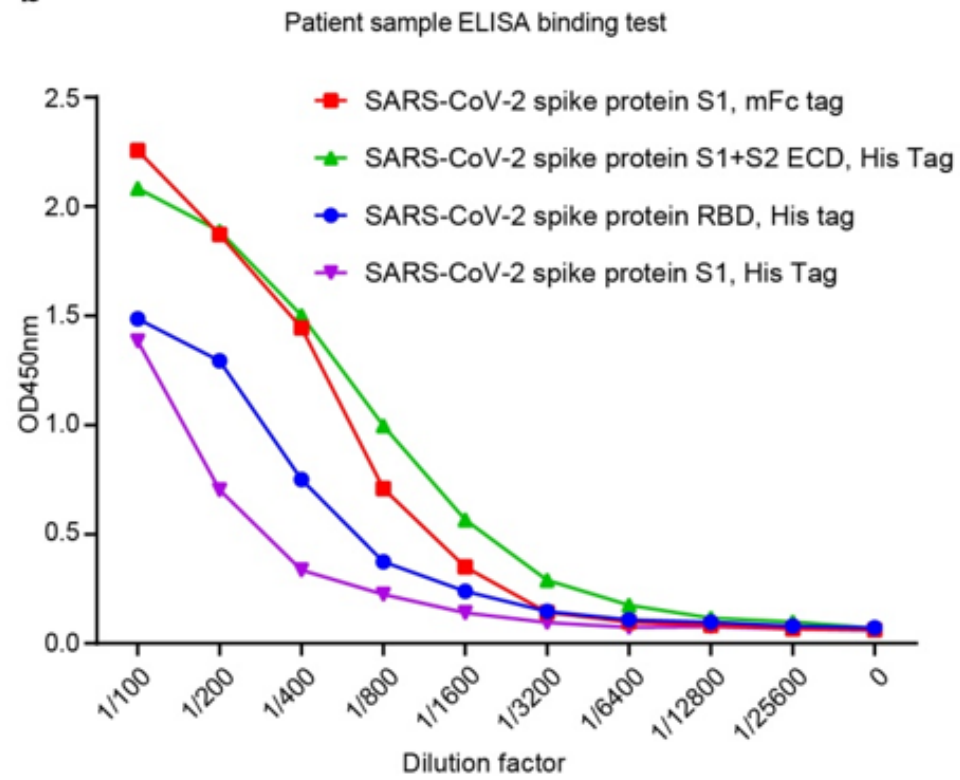

Figure 1

Analyses of plasma responses to SARS-CoV-2 proteins. (a) Serums from 23 convalescent patients and one healthy donor were analyzed for their binding abilities to the SARS-CoV-2 spike protein (Left) and nucleocapsid protein (Right) using ELISA. (b) Serum from a high-titer patient was further analyzed against full-length spike protein (S1+S2 ECD), S1-mFc fusion protein, S1-His protein and the S1 receptor binding domain (RBD) by ELISA. 

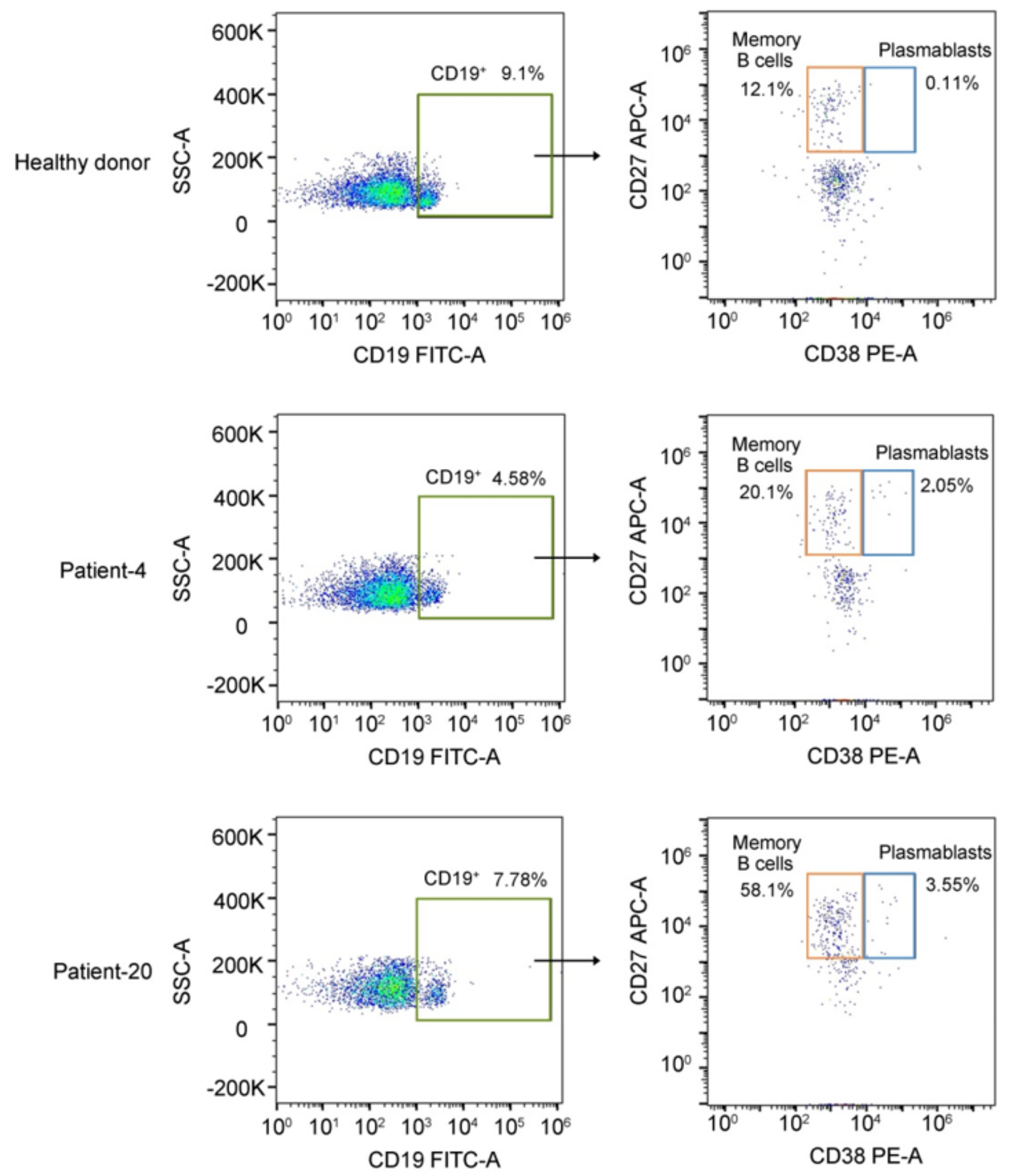

\section{Figure 2}

Immune profiling of B cells of COVID-19 patients by flow cytometry. PBMC cells from two convalescent patients and one healthy blood donor were stained by anti-CD19, CD27 and CD38 antibodies. Percentages of B cells (CD19+), memory B cells (CD19+CD27+CD38-) and plasmablast cells $(\mathrm{CD} 19+\mathrm{CD} 27+\mathrm{CD} 38+)$ in PBMC cells were analyzed by flow cytometry. 


\section{a}
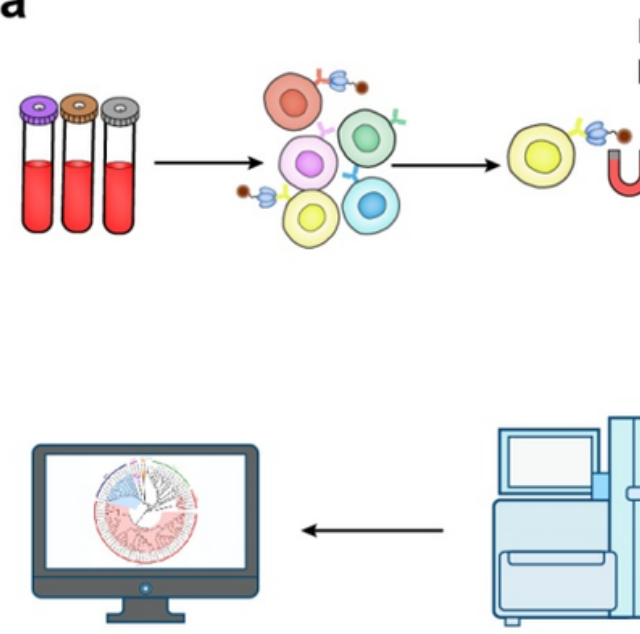

Lysis Buffer

Isolated
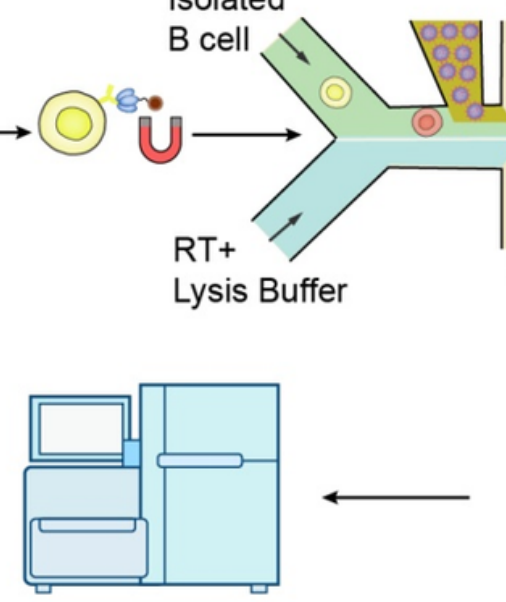

Oil

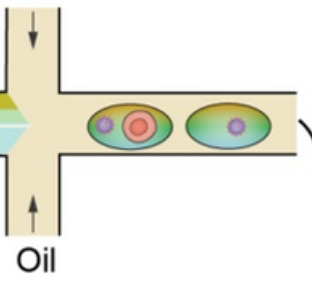

Oil

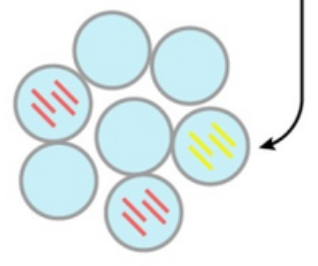

b

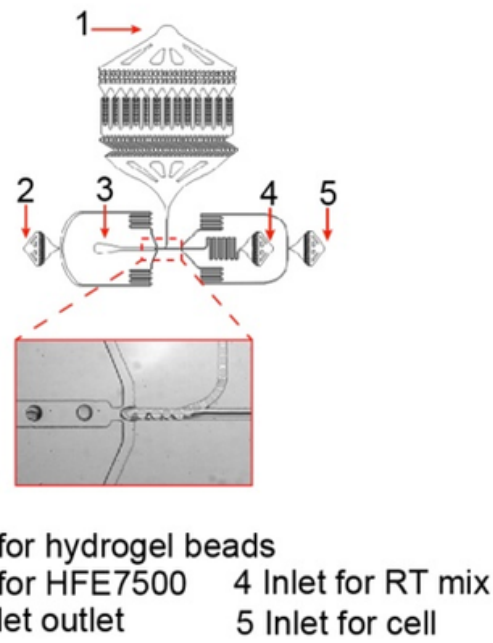

Figure 3

Identification of SARS-CoV-2 S protein specific antibodies from convalescent patients using single cell sequencing. (a) Schematic diagram of the antibody identification from convalescent patients using single cell sequencing. SARS-CoV-2 S protein binding B cells were isolated from PBMC of convalescent patients with magnetic beads that conjugated with biotinylated $S$ protein as probes. The isolated cells were individually compartmentalized in droplets along with lysis buffer, reverse transcriptase and one hydrogel bead. Each hydrogel bead carried VH and VL specific oligos tagged with a unique barcode. The resulting cDNAs from one cell carried the identical barcode. The barcoded cDNAs were sequenced to identify cognate heavy and light chain pairs. (b) The design of the microfluidics chip for cocompartmentalization of single cells and single hydrogel bead in droplets. 

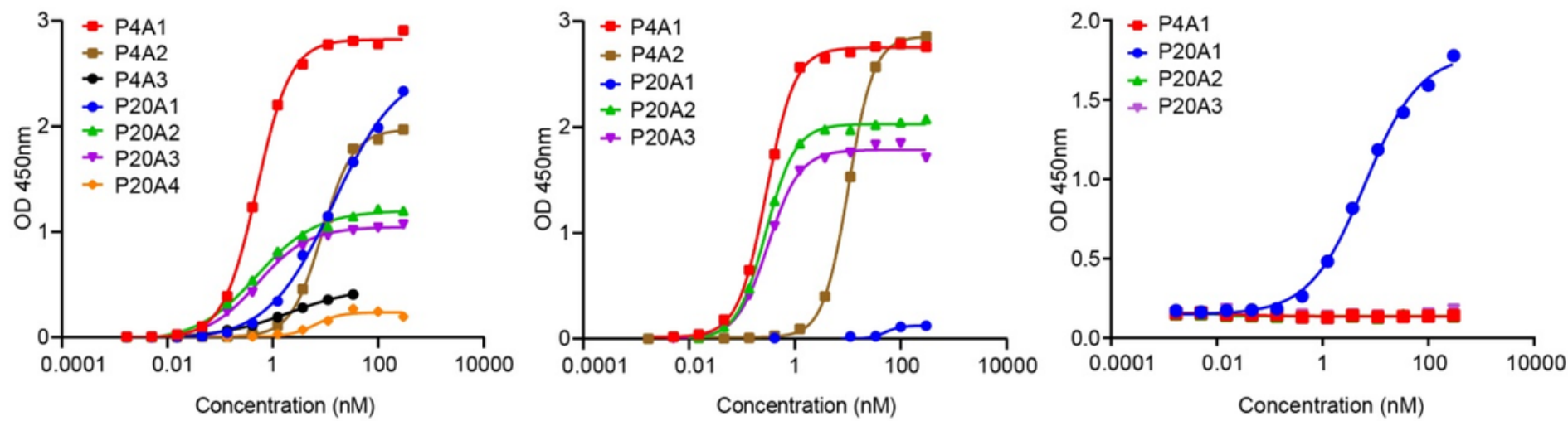

b

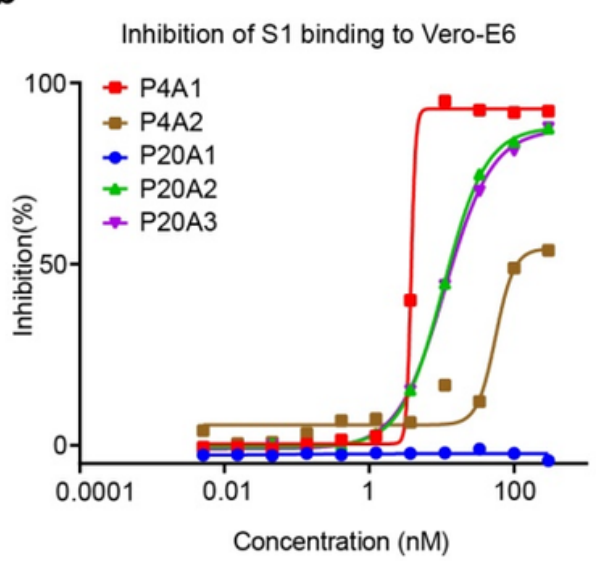

C

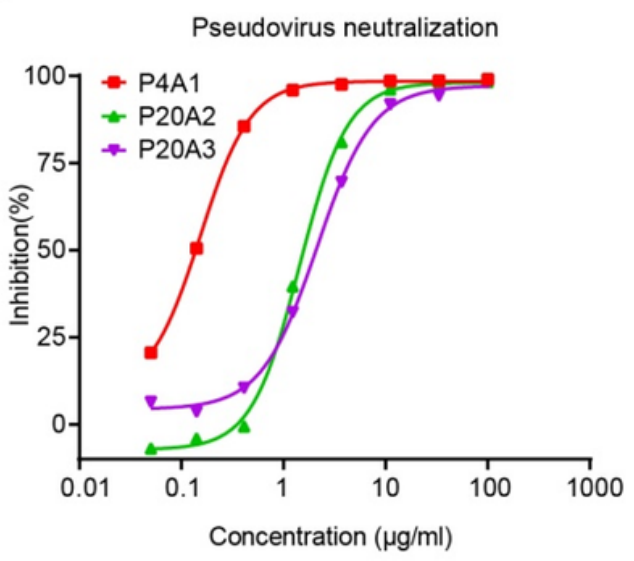

\section{Figure 4}

Characterization of SARS-CoV-2 S protein specific antibodies. (a) Binding of antibodies to the full length S protein(Left), S1 protein (Middle) and S2 protein (Right) were evaluated by ELISA. (b) Blockage of the binding of SARS-CoV-2 spike S1 protein to Vero E6 cell line by antibodies were evaluated by flow cytometry. Following the preincubation of spike S1-mFc fusion protein with serial dilution of antibodies, the antibody-S1 protein mixtures were added to the ACE2-expressing Vero E6 cells. The cells were stained with anti-mouse antibody and analyzed by flow cytometry. (c)Neutralization potency measured by SARSCoV-2 pseudovirus neutralization assay. Murine leukemia virus pseudotyped with SARS-CoV-2 S protein was incubated with serial dilution of antibodies. The mixtures were then added to ACE2 expressing HeLa cells. After $48 \mathrm{~h}$ incubation, neutralizations potency of antibodies was evaluated in a luciferase assay. 
a

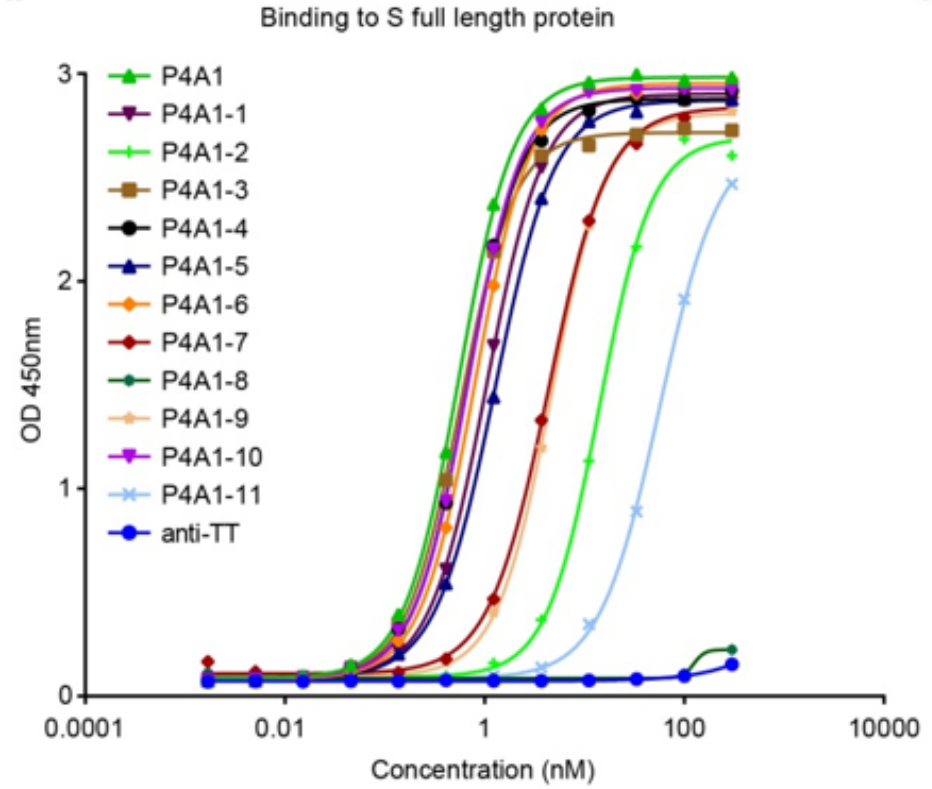

b

Blockage of the spike protein binding to ACE2

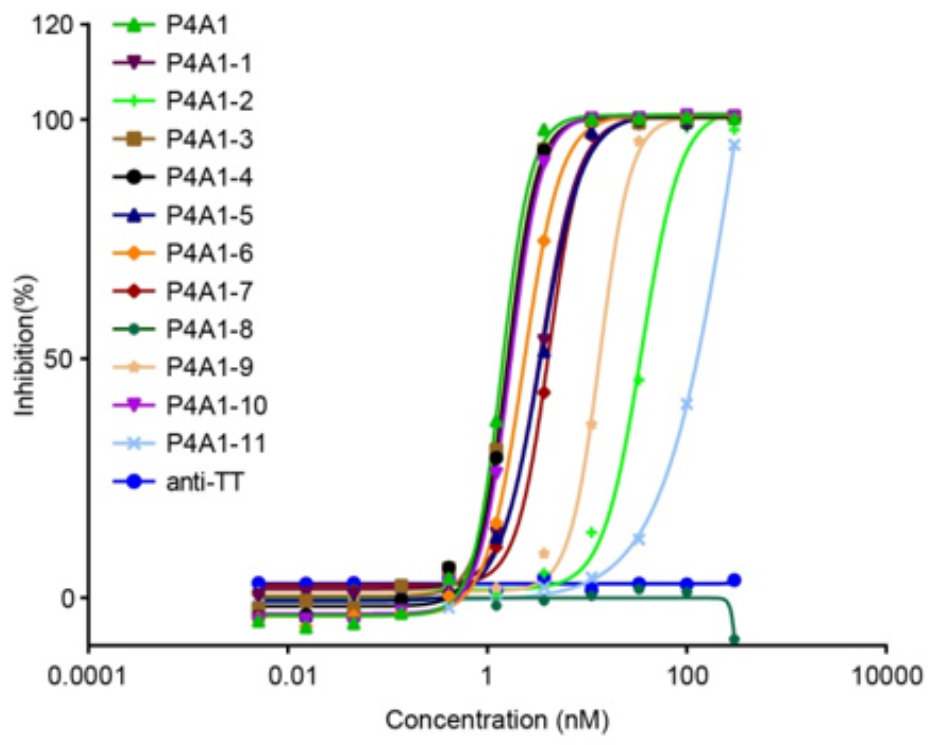

C

\begin{tabular}{|c|c|c|c|c|c|c|c|c|}
\hline & & 20 & CDR & & & & CDR2 & \\
\hline P4A1 & VQLVESGGG & L IQPGGSLRL & SCAASGFIVS & SNYMSW & QA & PGKGLEWVS I & IYSGGSTFYA & \\
\hline P4A1-3 & $\ldots \ldots \ldots$ & $\ldots \ldots \ldots$ & $\ldots$ & $\ldots$ & & $\ldots$ & $\ldots \ldots \ldots$ & \\
\hline P4A] & $\ldots \ldots \ldots$ & $\ldots \ldots \ldots$ & $\ldots$ & & & $\ldots \ldots$ & & \\
\hline $\mathbf{P}$ & $\ldots \ldots \ldots$ & $\ldots$ K. . . & $\ldots \ldots$ & . & $\ldots$ & $\ldots \ldots$ & $\ldots \ldots \ldots$ & \\
\hline & . & $\ldots \ldots$ & $\ldots$ & & $\cdots$ & & . & \\
\hline P4A & $\cdots$ & $\cdots$ & & & & & $\cdots$ & \\
\hline $\mathbf{P}$ & $\ldots \ldots \ldots$ & $\ldots \ldots$ & $\cdots$ & . & $\cdots$ & . & $\cdots$ & \\
\hline $\mathbf{P}$ & $\ldots$ T. & $\ldots \ldots$ & $\cdots$ & & & & . & \\
\hline & $\ldots \ldots \ldots$ & $\cdots$ & . & . & . & . & $\cdots$ & \\
\hline $\mathbf{P}$ & . & $\cdots$ & - & & & & & \\
\hline & $\ldots \ldots \ldots$ & & & & & & 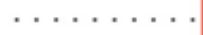 & \\
\hline 1 & $\cdots$ & . & $\ldots \ldots$ & $\cdots$ & $\cdots$ & . & $\ldots \ldots \ldots$ & \\
\hline
\end{tabular}

\begin{tabular}{l}
100 CDR3 \\
$I !$ \\
\hline
\end{tabular}

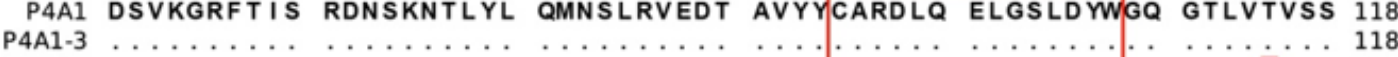

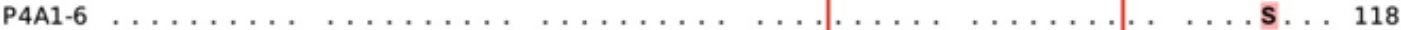

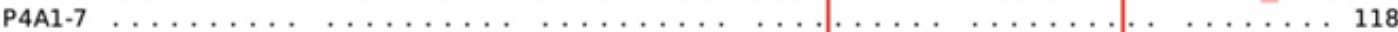

P4A1-8 $\ldots \ldots \ldots$

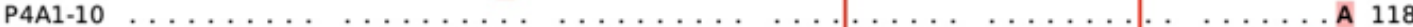

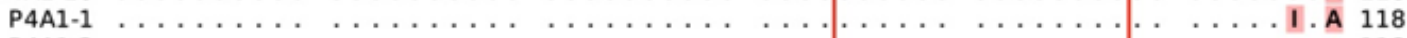

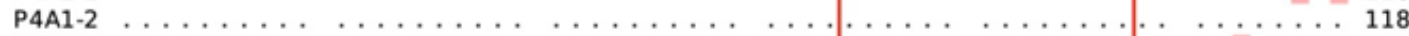

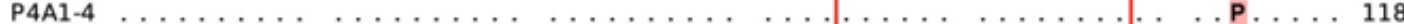

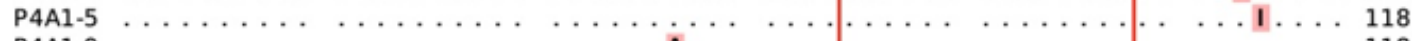

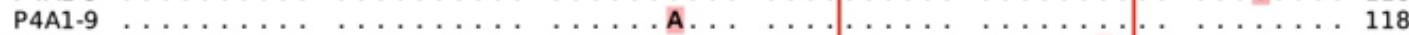

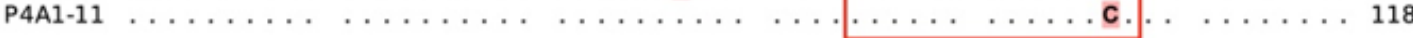

\section{Figure 5}

Identification and characterization of somatic variants of antibody P4A1. Eleven heavy chain sequences closely related to P4A1 were bioinformatically identified from NGS results. These P4A1-class heavy chains were reconstituted with the light chain from P4A1 and numbered P4A1-1 to P4A1-11. (a) Binding of the antibodies to the full length $S$ protein was evaluated by ELISA. (b) Blocking the binding of S1 protein to Vero E6 cell line by antibodies was evaluated by flow cytometry. (c) The amino acid sequences of the P4A1-class antibodies were aligned. 
a

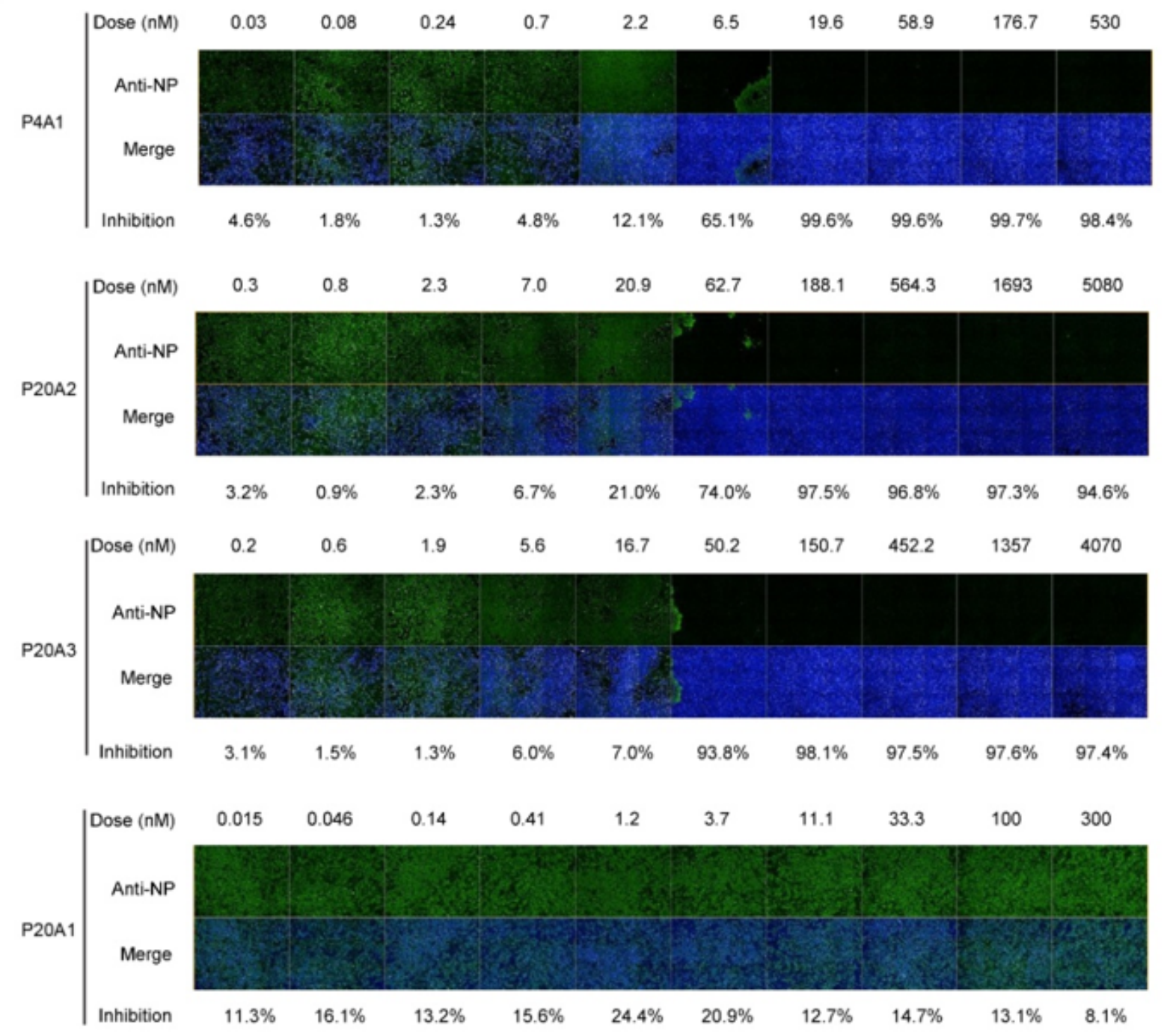

b
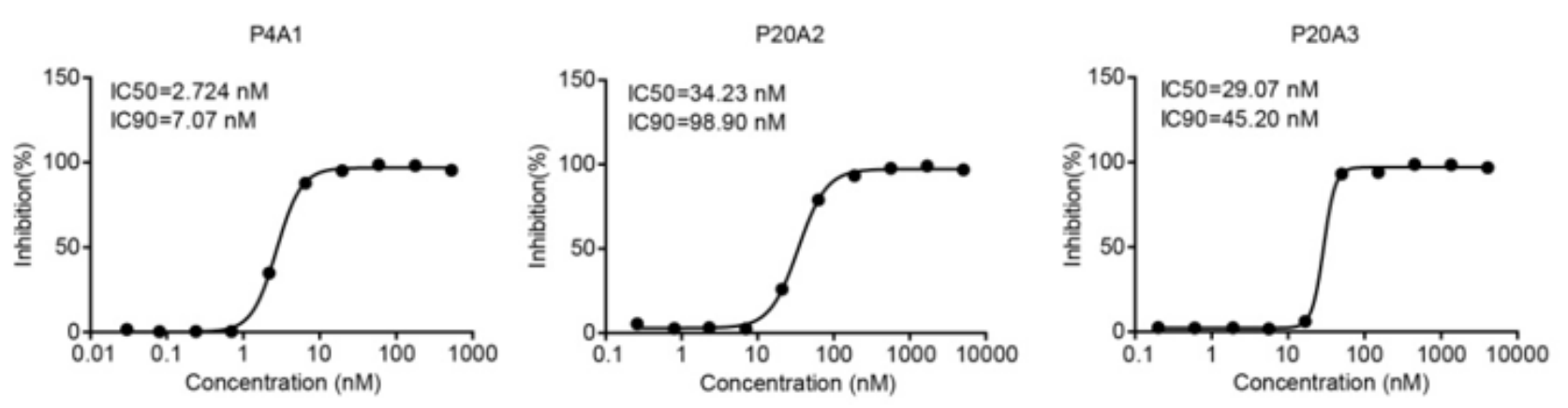

\section{Figure 6}

Antibody neutralization assay against SARS-CoV-2. Vero E6 cells were infected with SARS-CoV-2 virus at 100 TCID50 in the presence of antibodies. Cells were fixed, penetrated, and immunostained using antiSARS-CoV-2 NP rabbit serum. (a) Images of VeroE6 cells infected SARS-CoV-2 preincubated with antibodies of different concentrations. (b) Inhibition (IC50 and IC90) of antibodies were calculated using non-linear regression curve in Graphpad Prism 8.0. 

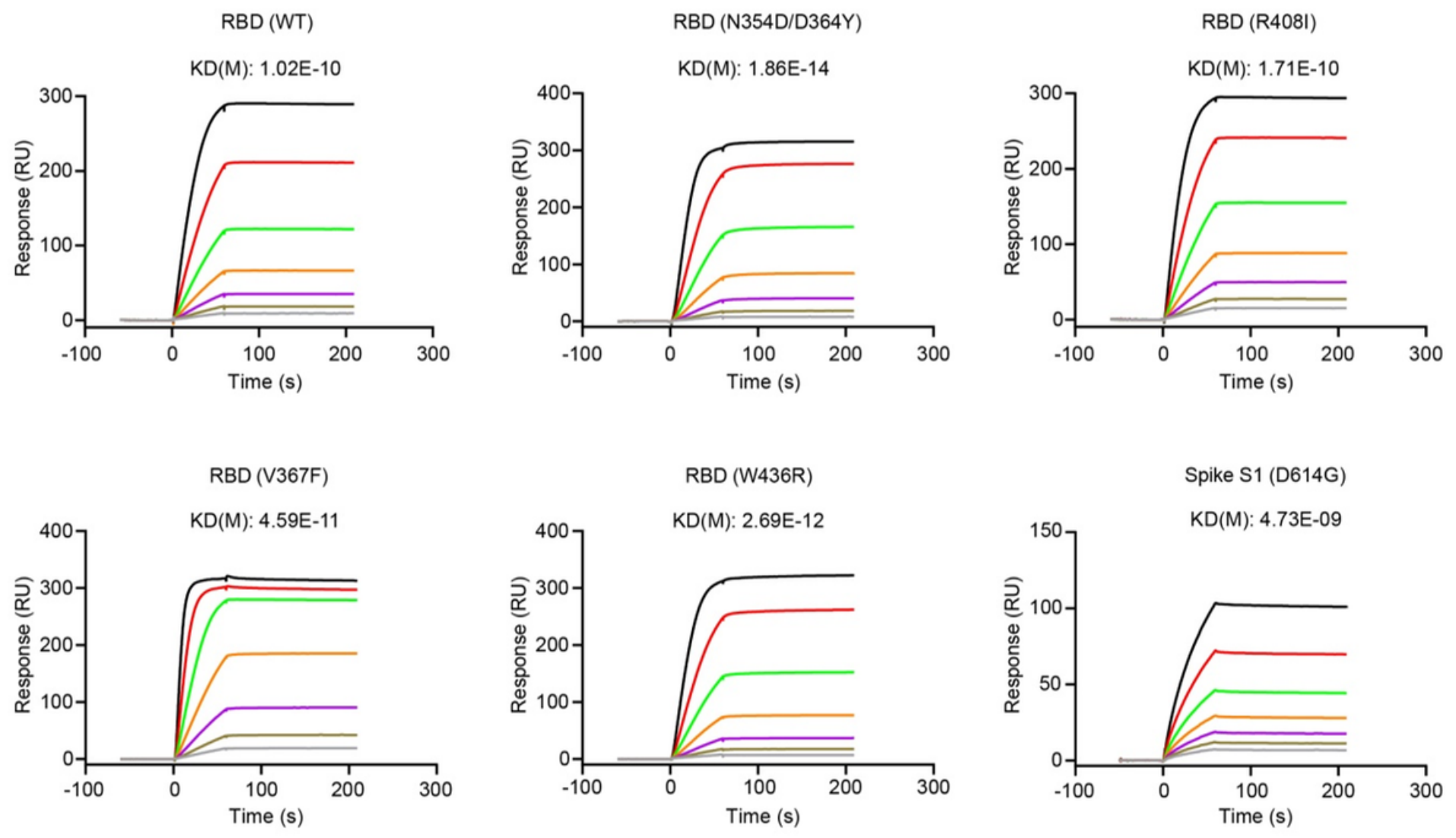

Figure 7

Binding of antibody P4A1 to SARS-CoV-2 S protein mutants determined by SPR. Antibody was immobilized onto a protein A sensor chip followed by injection of serially diluted SARS-CoV-2 wild-type RBD protein, RBD protein with N354D/D364Y, R408I, W436R, V367F mutation or spike S1 protein with D614G mutation. The Kd was calculated with the 1:1 binding model.

\section{Supplementary Files}

This is a list of supplementary files associated with this preprint. Click to download.

- supplementclinicalsignsSupplementaryTable1.xlsx

- hifibionankaiSARSCOV2neutralizingantibodyrawdata.zip 